\title{
The see-saw of wellbeing: What factors influence a learning advisor's wellbeing and happiness, in and outside of our work?
}

\author{
Lis Roche \\ Ara Institute of Canterbury, New Zealand \\ Email: lis.roche@ara.ac.nz
}

\begin{abstract}
Wellbeing is a commonly-used term — but what does it mean for tertiary learning advisors (TLAs), especially in the context of our professional practice? This paper reviews wellbeing literature in the disciplines of psychology, occupational health, and education, and discusses prominent models and definitions of both general and workplace wellbeing. It introduces a definition of wellbeing that proposes that individuals may manage and enhance their wellbeing by increasing or decreasing their psychological, physical and social resources and challenges, and relates this to the rewards and challenges of our TLA work, identified in recent TLA literature. This paper also examines the internal and external factors that influence an individual's wellbeing, such as their ways of coping with stress, personal traits, and personal resources and challenges, and suggests that individuals may manage and enhance their wellbeing by engaging in positive intentional activities of their choosing. While the wellbeing literature does offer some positive activities and strategies TLAs can use, there is a need for further research in the area of TLA wellbeing, particularly in relation to the emotional element of our TLA work.
\end{abstract}

Key words: tertiary education, learning advising, wellbeing

\section{Introduction}

The work of a tertiary learning advisor (TLA) can bring both satisfactions and rewards, as well as frustrations and challenges (Cameron, 2018; Malkin \& Chanock, 2018). In addition, the overall wellbeing of each of us is likely to be impacted by our interpretations of, and responses to, the nature and demands of TLA work. In what ways, then, does the wellbeing literature support TLAs in understanding the factors 
that influence our wellbeing, both in and outside of work? This paper discusses wellbeing theory both generally and in the workplace, and the related elements in TLA work. It examines various internal and external factors that may affect the equilibrium of an individual TLA's wellbeing, and introduces research about how individuals might increase their physical, psychological and social resources and enhance their wellbeing by undertaking intentional positive activities. This discussion of wellbeing is underpinned by a definition of wellbeing that has been recently proposed by researchers to help support self-management of wellbeing.

\section{Modelling, measuring and defining wellbeing}

'Wellbeing' is a widely used term that "concerns optimal experience and functioning" (Ryan \& Deci, 2001, p. 141). However, it is a topic of ongoing debate within the disciplines of psychology, occupational health, and education, amongst others. There appears to be little consensus on the measurement of wellbeing and no universally agreed definition (for reviews, see Cooke, Melchert, \& Connor, 2016; Dodge, Daly, Huyton, \& Sanders, 2012; Lent, 2004; Ryan \& Deci, 2001). In fact, as Dodge et al. (2012) point out, there is even disagreement on how wellbeing should be spelled.

The existing wellbeing research offers a broad range of wellbeing models and related terminology. The two most influential approaches to understanding wellbeing appear to be 'hedonic' and 'eudaimonic' wellbeing, which stem from the disciplines of psychology and sociology (Cooke et al., 2016). Hedonic wellbeing deals with pleasure and happiness, and is informed by ancient hedonism, the balance of pleasure over pain. The most prominent hedonic model is concerned with subjective wellbeing (Diener, 1984), and focuses on happiness and life satisfaction. The other key approach, eudaimonic wellbeing, encompasses Aristotle's view of virtuous living and reaching one's potential. One of the most common eudaimonic theories is psychological wellbeing (Ryff, 1989), which focuses on thriving, or psychological flourishing.

However, "despite the differences in approach, most researchers now believe that wellbeing is a multi-dimensional construct" (Dodge et al., 2012, p. 223); that is, that 
subjective and psychological wellbeing and their many facets may fit under one conceptual framework. For example, Seligman's (2011) recent 'Flourish' theory of wellbeing, adopted by the positive psychology movement, integrates components of both subjective and psychological wellbeing. According to Seligman, positive emotion, engagement, relationships, meaning, and accomplishment (PERMA) are the building blocks to wellbeing, and when combined these components enable human flourishing - and flourishing is, Seligman believes, "the gold standard for measuring well-being" (p. 13).

The measurement of wellbeing is, like its modelling, approached in various ways. For example, subjective wellbeing is measured by self-report, with individuals indicating the presence of positive affect, the absence of negative affect, and life satisfaction, while psychological wellbeing is measured by both self-report and external judgments of thriving and positive psychological functioning, or meaning in life. Wellbeing is often measured over one of three timeframes: immediate/momentary positive or negative thoughts/emotions; daily or recent satisfaction or emotion/affect; or overall life satisfaction, dispositional affect, or sense of purpose (Grawitch, Ballard, \& Erb, 2017; Lent, 2004). Moreover, wellbeing is measured globally (context-free) or in specific contexts, such as in work, family, or social relationships (Lent, 2004).

It is clear, then, that the topic of wellbeing has its ambiguities. As a consequence, Dodge et al. (2012) believe that a clear definition of wellbeing is neededparticularly as a basis for measuring wellbeing quantitatively. They argue that so far researchers have not yet successfully defined wellbeing, and have merely described or focused on dimensions of wellbeing (positive and negative affect, happiness, life satisfaction, ability to fulfil goals, quality of life, positive functioning, flourishing etc.). To address this deficit, Dodge et al. propose their dynamic equilibrium definition, based on Headey and Wearing's 'set-point' theory (1989, 1991, 1992, as cited in Dodge et al., 2012), and Hendry and Kloep's (2002) lifespan model of development. Dodge et al.'s definition uses the image of a see-saw to represent 
wellbeing as "the balance point between an individual's resource pool and the challenges faced" (p. 230) (see Fig. 1), and they propose that "stable wellbeing is when individuals have the psychological, social and physical resources they need to meet a particular psychological, social and/or physical challenge. When individuals have more challenges than resources, the see-saw dips, along with their wellbeing, and vice-versa" (p. 230):

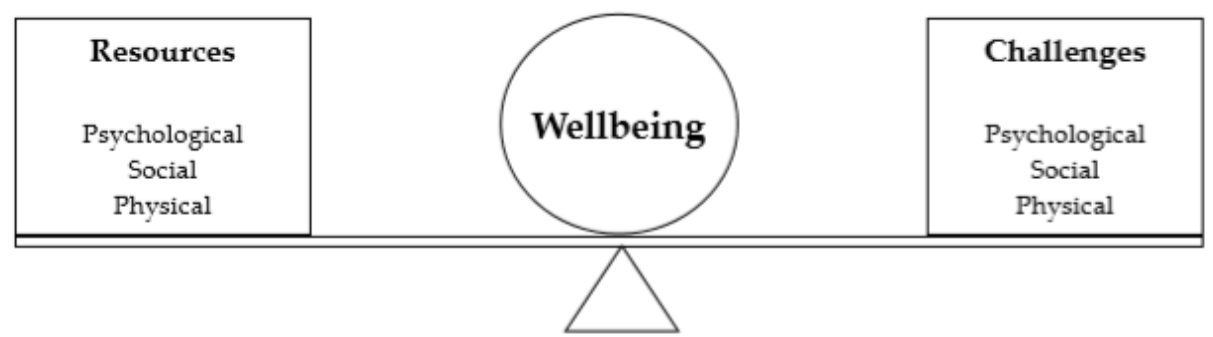

Figure 1. Definition of wellbeing (Dodge et al., 2012, p. 230). Reproduced with permission from Rachel Dodge.

This definition of wellbeing by Dodge et al. (2012) presents a simple yet precise model; has universal application across a wide range of settings, regardless of people's age, culture and gender; and is optimistic as it supports self-management of wellbeing. Given this, Dodge et al.'s definition has the potential to assist each TLA with the selfmanagement of their overall wellbeing. Furthermore, the dynamic equilibrium concept underlying Dodge et al.'s definition aligns with the balance concepts used in the various models of workplace stress and wellbeing, which may also support TLAs of gaining a more in-depth understanding of managing their individual and collective wellbeing, both in and outside of work.

\section{Wellbeing in the workplace}

According to Leiter and Cooper (2017), workplace wellbeing "encompasses physical health and comfort, mental health, a preponderance of positive over negative effect, and positive attitudes towards work" (pp. 1-2). There is much discussion in current occupational health literature about who should take responsibility for employee 
workplace wellbeing being. For instance, the improvement of the health and wellbeing of employees has become a priority for many organisations - either to improve productivity or in recognition that organisations have a responsibility for employee wellbeing (Kelloway, 2017). However, the literature also acknowledges that employees have a responsibility to contribute to their workplace wellbeing by making the most of workplace resources; that is, "to develop the skills, attitudes, and inclinations to make wellbeing a core personal value as well as a corporate value" (Leiter \& Cooper, 2017, p. 2).

In prominent models of workplace wellbeing, the balance of job characteristics is the primary determinant of employee stress or wellbeing. For example, employees might measure their workplace stress/wellbeing by their perception of whether the effort they expend in meeting their work demands/obligations is reciprocated by the rewards of adequate pay, esteem, and promotion/job security (Siegrist's EffortReward Imbalance (ERI) model, 1996); or whether their 'job resources' (such as collegial support, performance feedback, pay, skill variety, job control, career opportunities, role clarity, autonomy, job security) are sufficiently balanced against their 'job demands' (such as high work pressure, future job insecurity, emotionally demanding client interactions, workplace conflicts), as suggested by the Job Demands-Resources [J-DR] model: Demerouti, Bakker, Nachreiner, \& Schaufeli, 2001; Schaufeli \& Bakker, 2004). Both the ERI and J-DR models reflect the workrelated satisfactions, key rewards, frustrations and challenges that have been reported in recent surveys of TLAs in New Zealand (Cameron, 2018) and Australia (Malkin \& Chanock, 2018), outlined in the following section.

Importantly, an employee's workplace wellbeing has a strong influence on their wellbeing in other parts of life, and vice versa (Grawitch et al., 2017; Warr, 1999). This means that work incidents or conditions that are very stressful to one individual may be less stressful, or even motivating, to another (Manthei, 1997). In addition, some workplace stress/wellbeing models acknowledge the non-work factors that affect an individual's workplace wellbeing. For example, an important addition to the JD-R model's resources is the recognition that both an employee's job resources and 'personal resources' (that is, certain malleable personality characteristics, such as 
self-efficacy, organisational-based self-esteem, and optimism) contribute to their work engagement and help to determine their wellbeing at work (Xanthopoulou, Bakker, Demerouti \& Schaufeli, 2009). Moreover, Xanthopoulou et al. (2009) identify these personal resources as important for both work-related wellbeing and general psychological wellbeing. Also, according to Grawitch et al.'s (2017) Dynamic Model of Workplace Wellbeing, an individual's wellbeing in the workplace depends on how they allocate their personal resources of time, energy and money, as well as their external resources (e.g. both non-work and workplace supports). Furthermore, Grawitch et al. contend, individual differences (such as personality traits, attitudes/interests, demographics, marital/parental status, health, competencies etc.) can facilitate or obstruct an individual's use of their personal and external resources. Therefore, for example, a TLA who is a parent or has ill health may at times have less time and/or energy to allocate to their work, or to access their social supports. This is highly relevant when considering TLA wellbeing, particularly in light of the emotional element of our role in student support.

\section{Wellbeing of tertiary learning advisors}

Individual TLAs will be aware that our work, particularly our role in student support, may impact on our overall wellbeing. Yet, a comprehensive search of the global TLA literature found no specific studies of TLAs' wellbeing, per se. Helpfully, however, recent surveys of TLAs in New Zealand (Cameron, 2018) and Australia (Malkin \& Chanock, 2018) have reported TLAs' work-related satisfactions, key rewards, frustrations and challenges. These survey results aid in informing the topic of TLA wellbeing, as does the literature that explores the emotional dimensions of our TLA work and student support in higher education.

Both New Zealand and Australian surveys report that TLAs have ongoing and increasing work stressors, such as marginalisation of the TLA role; job insecurity; and work intensification, including reduced staffing resource and time. Accordingly, as Cameron (2018) notes, organisational changes to learning services mean TLAs cannot provide the service to students that we aspire to (personal communication, 
June 19, 2018). This challenge is not new: in their study about stress associated with changes in the tertiary education sector, Thomas and Bennett (2002) reported that reduced staffing, increased student contact hours, and insufficient time for intensive support for an increasingly diverse student population all caused significant stress for TLAs. TLAs further report that our role, and/or what we can achieve in our role, is not always understood or valued by managers, academic staff, and students (Cameron, 2018; Malkin \& Chanock, 2018; Thomas \& Bennett; 2002). As such, it could be argued that this lack of understanding contributes to other identified issues, such as increased workload and job security.

However, the recent surveys also show that despite these challenges, many of us are highly satisfied in our TLA roles. Our key rewards relate to two areas: work environment and culture (autonomy, variety and flexibility of the work, and collegiality and relationships with peers), and student interaction (face-to-face work with students, particularly one-to-one and with small groups; helping people learn; seeing the impact of our work; and knowing, albeit non-quantifiably, that we are making a difference for students) (Cameron, 2018; Malkin \& Chanock, 2018). Using Dodge et al.'s (2012) wellbeing model, TLAs might consider each of these rewards as 'resources' in our wellbeing. In particular, most TLAs feel that student interaction is a supremely rewarding element of our role (Cameron, 2018; Malkin \& Chanock, 2018). However, the intensification of student engagement, with increasingly high levels of student need, has its costs for TLAs' wellbeing. Consequently, these 'good' parts of the job may also contribute to the 'see-saw' rise-and-fall experience in our TLA work.

TLAs might consider the emotional element of our work, in particular, as both a resource and a challenge for our wellbeing. Students experience an emotional journey as they enter and acclimatise to tertiary study (Beard, Clegg \& Smith, 2007); accordingly, as Mitchell (2008) notes, TLAs experience both positive and negative emotionally charged learning interactions when working with students - particularly in one-to-one consultations when students share their emotional responses to their learning. As Carter and Bartlett-Trafford (2008) identify, the "fostering, guiding and psychological support [te aroha]" element of TLA practice brings depth of meaning 
to our work (pp. 53-54); thus, TLAs might consider it a wellbeing 'resource'. However, as Cameron (2018) cautions, the positive effects of such intrinsic motivators depend "on the balance between the rewards of the role and the inevitable frustrations" (p. 59), particularly given the current economic pressures that are dictating reduction or changes in TLAs' one-to-one work with students. For example, in some institutions, those individual consultations are on a purely highneeds basis: if a TLA is working in an environment of reduced staffing resource and an increasingly high level of student need, the emotional element of our work with students may become a challenge, rather than a resource, and contribute to tipping our 'see-saw' of wellbeing. This is particularly relevant given recent reports that students are experiencing considerable levels of stress, anxiety and depression (New Zealand Union of Students' Associations, 2018) and academic anxiety (Sotardi \& Friesen, 2017).

With this in mind, it is surprising that the emotional dimensions of our TLA work are not more significantly reflected in the literature on TLA practice. Apart from Mitchell's (2008) discussion of the skills and competencies required to engage with and respond to learners' emotions, the most relevant article related to TLAs' own emotions is from Huyton (2009) — who, notably, is an author of Dodge et al.'s (2012) article defining wellbeing. Huyton (2009) voices particular concern about the 'emotion work' (management of emotions) of higher education practitioners involved in student support and learning development—particularly in light of the work intensification in those roles, and especially for those practitioners who have little or no previous experience of emotion work. Huyton considers how work intensification and performance requirements can result in practitioners feeling overwhelmed and in need of support. Furthermore, Huyton explains that these challenges make it hard for practitioners to sustain an authentic emotional display with students. She relates this to the concept of 'emotional labour', first introduced by Hochschild (1983) to explain the labour required to control emotions in a range of workplace contexts, by either suppressing or modifying expressions of feelings ('surface acting'), or manipulating thoughts and feelings, for example, by self-talk or taking perspective ('deep acting'). Huyton notes that emotional labour can have damaging effects on both the quality of the practitioner's student support, and their own wellbeing. This point is reflected in 
the discussion of the emotional aspects of teaching practice in the wider educational literature, where emotional labour is said to result in educator stress and exhaustion; for example, Hargreaves' (1998) discussion of the role of emotions in teaching. Furthermore, O’Toole, Ogier-Price, and Hucks' (2010) exploration of New Zealand tertiary teachers' emotion in their teaching contexts acknowledged that while teachers are committed to managing their emotions professionally, their pretence or suppression of certain emotions can result in emotional exhaustion. Therefore, as Huyton's paper concludes, higher education institutions need to recognise both the value of emotion work and its impact on tutors in their learning relationships with students, and as employers, provide appropriate staff support and development. Learning advisor and other related literature, therefore, identifies many of the workrelated rewards and challenges experienced by TLAs. In reference to Dodge et al.'s (2012) definition of wellbeing, these identified rewards and challenges could be argued to contribute to our individual psychological, social and physical resources and challenges, and affect the equilibrium of our wellbeing. However, these work factors are not the only factors influencing a TLA's wellbeing: we should also consider the personal dispositions and history we each bring to the workplace, as our individuality is likely to colour how we interpret the challenges and rewards of our TLA work, and the intensity of our work experiences.

\section{Factors influencing an individual TLA's wellbeing}

An individual's ways of coping with stress is an important wellbeing factor. For example, some incidents or environments, such as those related to work intensification, may expose individual TLAs to stresses we feel we cannot currently manage, while others consider those situations routine, or a positive challenge. Therefore, an individual's behavioural, emotional and cognitive ways of coping with stress may be "organized, flexible, and constructive... [or involve] the repeated use of rigid, disorganized, or derogatory ways of coping [which] is diagnostic of exposure to unmanageable levels of stress" (Skinner, Edge, Altman \& Sherwood, 2003, p. 231). Skinner et al.'s (2003) meta-analysis of the ways of coping identified 400 ways of 
coping, which are classified these into 13 'families' of coping. Of those, five core categories are identified as: problem solving, support seeking, escape-avoidance, distraction, and positive cognitive restructuring (such as seeing the situation differently and more positively). Further categories of ways of coping included rumination, helplessness, social withdrawal, emotional regulation, information seeking, negotiation, opposition, and delegation. Whether a pattern of coping is characterised as good/bad, healthy/unhealthy, constructive/harmful comes down to "whether the individual can handle the demand or is overwhelmed by it" (Skinner et al., 2003, p. 231). Moreover, an individual's responses to stress, their ways of coping, and their experience of the manageability or unmanageability of stress may change over a situation if pressure on a person increases or decreases, or change over time, especially as they age and develop. Our response to our TLA-related challenges, then, will depend on our individual evaluation of the demands of a situation, our low or high personal resources, and/or the sufficiency of our social supports. In light of Dodge et al.'s (2012) dynamic equilibrium model of wellbeing, it could be argued that our intentional use of "organized, flexible, and constructive" ways of coping (Skinner et al., 2003, p. 231), both outside of and at work, might employ (or even add to) our psychological, social or physical resources, and thus support or retain the balance of our 'see-saw' of wellbeing.

Other factors that influence an individual's wellbeing are stable personality traits and characteristics. For example, a person's predisposition to experience positive or negative emotional states has been identified as being directly related to their levels of happiness and wellbeing (Diener, 1984, cf. Bradburn. 1969). Therefore, Diener (1984) contends, if an individual has general positivity (a 'sunny' disposition), their temperament predisposes them to make globally positive perceptions of events or environments. Furthermore, the personality trait of dispositional affectivity has a significant effect on people's work attitudes, workplace experiences, and work behaviour (Ng \& Sorensen, 2009), as well as on how people remember and report their levels of job satisfaction or dissatisfaction (Staw \& Cohen-Charash, 2005). Wellbeing has been linked to other personality traits, including the Big Five personality traits of extraversion, agreeableness, conscientiousness, openness to experience, and emotional stability (neuroticism) (McCrae \& Costa, 2003). While 
personality traits and characteristics are considered stable, Lent (2004) suggests individuals can be supported through counselling psychology to develop cognitive, behavioural and social resources that may function alongside (and, notably, "possibly ... compensate for") personality traits (p. 498). These resources include mental activities, such as learning to set goals, use positive ways of coping, manage negative affect, or develop self-efficacy; physical activities; and social activities, such as building caring relationships and social support. These types of activities are known as positive psychological interventions, which is "an umbrella term used to describe a treatment method or activity designed to foster happiness" as part of a randomised controlled intervention (Layous, 2018, p. 7).

Therefore, each TLA's ways of coping with stress, and personality traits or characteristics, should be identified as factors of individual wellbeing. Also, in reference to Dodge et al.'s (2012) dynamic equilibrium definition, we might consider these ways of coping and personal traits as resources or challenges, or as contributing to how we each manage our work-related resources and challenges. While Dodge et al. do not provide examples of specific resources, these are discussed by Hendry and Kloep (2002), whose work informs the resource/challenge element of Dodge et al.'s definition of wellbeing. Hendry and Kloep suggest each individual has a pool of potential resources to help them cope with challenges that arise, and that each individual's resource system will differ. Therefore, an individual's resources will be a factor in their wellbeing. Some resources will be innate "biological dispositions (e.g. genetics, health, 'personality')", while some are determined by culture or class, and some are learned, such as "social resources; skills in various domains; [and] selfefficacy" (Hendry \& Kloep, 2002, p. 19). Similarly, Lyubomirsky, Sheldon, and Schkade's (2005) integrated model of happiness shows that an individual's wellbeing and happiness are influenced by genetic, circumstantial and dynamic factors. Using research from the wellbeing literature, Lyubomirsky et al., identify the variance of each of these factors of wellbeing. For example, they identify that approximately 50 percent of wellbeing is accounted for by a 'genetic set point'. This genetic factor, which by definition is immutable, relates to personality traits and cognitive outlooks, and appears to correspond with part of what Hendry and Kloep call 'biological dispositions'. Further, Lyubomirsky et al. identify that 'circumstances' (such as 
demographics, nationality and culture, life events, and life status variables - that is, Hendry and Kloep's 'structural resources') account for 10 percent of the population variation in happiness and wellbeing. Lyubomirsky et al. also suggest that while circumstances can be changed short-term, changes in circumstances have limited potential for long-term and sustainable changes in happiness and wellbeing.

Notably, the remaining 40 percent of the population variation in wellbeing - which correspond to Hendry and Kloep's 'learned' resources - can, Lyubomirsky et al. (2005) propose, be significantly influenced by 'intentional activity'. That is, by an individual's effortful engagement in "discrete actions or practices" (p. 118). Unlike Lent's (2004) focus on positive psychological interventions used in counselling psychology, Lyubomirsky et al. (2005) focus on intentional 'positive activities' or happiness-increasing activities that individuals can undertake independently. These include:

behavioral activity, such as exercising regularly or trying to be kind to others; ;.. cognitive activity, such as reframing situations in a more positive light or pausing to count one's blessings;... and some kinds of volitional activity, such as striving for important personal goals... or devoting effort to meaningful causes (p. 118).

Moreover, meta-analyses of studies of positive psychology interventions have shown that increasing positive emotions, positive thoughts/cognitions, or positive behaviours will increase subjective wellbeing and psychological wellbeing (Bolier et al., 2013; Sin \& Lyubomirsky, 2009). This idea of actively intervening in one's wellbeing clearly links to Dodge et al.'s (2012) dynamic equilibrium definition of wellbeing and their proposal that individuals can self-manage their wellbeing by building their psychological, physical, and social resources - and it is this factor that each TLA can actively act upon to manage and stabilise our wellbeing, in and outside of work.

However, it is important that each of us chooses the type and timing of our intentional activities. According to Layous (2018) and Lyubomirsky and Layous (2013), the effectiveness of intentional positive activities depends on several elements, such as: the type of activity, how often it is done, and variety; how engaged the person is in the activity (for example, is motivated, feels supported); and, most importantly, the person-activity fit. This last element depends on a person's 
personality, culture, affective state, age, etc., and is evidenced by how often the person completes an activity and their subsequent level of happiness. Person-activity fit is especially important for sustainable changes in levels of happiness and wellbeing (Layous, 2018) - therefore, each TLA should choose activities that appeal deeply to us and, equally, support our colleagues, friends and family in their respective choices.

Unfortunately, the current body of wellbeing literature offers fewer than expected strategies that TLAs might use as intentional positive activities at work or outside of work. This is surprising, given the considerable discussion about wellbeing, stress and burnout in the various disciplines - including our own - and the conclusions of many authors that individuals can contribute to their wellbeing. Most of the ideas offered by the wellbeing literature will not be new to most TLA practitioners, as they reflect the ideas we commonly discuss with students to manage wellbeing and prevent anxiety. These include journal writing or expressive writing; building strong social relationships both at work and outside of work; mental detachment from work; taking work breaks and lunch breaks, including leaving the building; learning mindfulness techniques; reflection on practice; informal peer discussion; peer supervision; and keeping an emotion diary. There are also numerous wellbeing activities suggested outside of academic literature; while these are not all necessarily grounded in research, TLAs may find them of interest, for example,

- activities suggested by the Action for Happiness movement (https://www.actionforhappiness.org/take-action) and

- the Mental Health Foundation of New Zealand (https://www.mentalhealth.org.nz/home/ways-to-wellbeing/ and https://www.mentalhealth.org.nz/home/our-work/category/44/working-wellguide-and-resources).

A number of the ideas and activities suggested in the wellbeing literature warrant further research for how they might specifically support TLAs' wellbeing-for example, research could explore individual TLAs' own emotional, behavioural and cognitive ways of coping, and our social and individual supports. Also, in light of the emotion work in our TLA role, and especially given the reported increases in student 
stress and anxiety, there is an argument for extending Huyton's (2009) discussion of the emotional labour involved in student support and learning development. This could include an exploration of how using emotion regulation strategies to decrease negative emotions or increase positive emotions might support how we manage our experiences or express our emotions in our TLA work. While the wider education literature has explored the role of teachers' emotions and the regulation of emotions (for example, Hargraves, 1998; O'Toole et al., 2010; Sutton, 2004), emotion regulation does not appear to have been discussed in TLA literature. Perhaps TLAs can make extrapolations from the education literature, but the (often intense) one-toone elements of our TLA role and the experiences and strategies related to the emotional element of our work may differ from the emotional experiences of classroom teachers.

\section{Conclusion}

According to workplace wellbeing models, the balance of job and personal resources is key to managing workplace challenges. This paper introduces TLAs to a definition of wellbeing that uses the concept of dynamic equilibrium, or a see-saw, to explain how individuals balance their psychological, physical and social resources and challenges that are necessary for maintaining stable wellbeing (Dodge et al., 2012). This definition is particularly relevant in light of the results of recent surveys (Cameron, 2018; Malkin \& Chanock, 2018), where TLAs report high levels of satisfaction in our work, but also show that the intensification of student engagement and increased levels of student stress contribute to our work challenges. Furthermore, this paper suggests that the emotional elements of our TLA student support role, as discussed in other TLA literature, are both a resource and challenge for TLA wellbeing. As Edelwich and Brodsky (1980) emphasise in their seminal work on burnout in the helping professions, "for most people a balanced life is the most important intervention against Burn-out that there can be" (p. 230). Therefore, Dodge et al.'s (2012) definition has the potential to support individual TLAs to selfmanage our wellbeing, both at and outside of work. 
The literature on wellbeing and happiness shows that TLAs might manage the equilibrium of our personal and workplace wellbeing — and even enhance our wellbeing - when we learn positive ways of coping with stress and use positive psychological interventions, and, in particular, engage independently in positive intentional activities of our own choosing. However, there is limited work on what those positive intentional activities might be. Hence, further research is needed in the area of TLA wellbeing, particularly in the area of positive activities that TLAs might choose to engage in to support and manage the intensity of student interactions. Such TLA-specific wellbeing literature would benefit both the TLA profession and practitioners alike, supporting our agency in fostering collective and individual wellbeing. 


\section{References}

Beard, C., Clegg, S., \& Smith, K. (2007). Acknowledging the affective in higher education. British Educational Research Journal, 33(2), 235-252.

Bolier, L., Haverman, M., Westerhof, G. J., Riper, H., Smit, F., \& Bohlmeijer, E. (2013). Positive psychology interventions: A meta-analysis of randomized controlled studies. BMC Public Health, 13, 119. doi: 10.1186/1471-2458-13-119

Cameron, C. (2018). Tertiary learning advisors in Aotearoa/New Zealand: Part three: Why do we stay?: Rewards and challenges. ATLAANZ Journal, 3 (Special Issue: Identity and Opportunity): 44-66.

Carter, S. \& Bartlett-Trafford, J. (2008). Who are we? Aotearoa New Zealand Tertiary learning advisors talk about themselves. In E. Manalo, J. Bartlett-Trafford, \& S. Crozier (Eds.), Walking a tightrope - the balancing act of learning advising: Refereed proceedings of the 2007 Association of Tertiary Learning Advisors of Aotearoa New Zealand (ATLAANZ) Conference (pp. 39-61). Auckland, New Zealand: ATLAANZ.

Cooke, P. J., Melchert, T. P., \& Connor, K. (2016). Measuring well-being. The Counseling Psychologist, 44(5), 730-757. doi:10.1177/0011000016633507

Demerouti E., Bakker, A. B., Nachreiner, F., \& Schaufeli, W. B. (2001). The Job DemandsResources model of burnout. Journal of Applied Psychology, 86, 499-512.

Diener, E. (1984). Subjective well-being. Psychological Bulletin, 95(3), 542-575. doi:10.1037/0033-2909.95.3.542

Dodge, R., Daly, A., Huyton, J., \& Sanders, L. (2012). The challenge of defining wellbeing. International Journal of Wellbeing, 2(3), 222-235. doi:10.5502/ijw.v2i3.4

Edelwich, J., \& Brodsky, A. (1980). Burn-out: Stages of disillusionment in the helping professions. New York, NY: Human Sciences Press.

Grawitch, M. J., Ballard, D. W., \& Erb, K. R. (2017). Work and wellbeing: Creating a psychologically healthy workplace. In C. L. Cooper \& M. P. Leiter (Eds.), The Routledge companion to wellbeing at work (pp. 249-268). New York, NY: Routledge.

Hargraves, A. (1998). The emotional practice of teaching. Teaching and Teacher Education, 14(8), 835-854.

Hendry, L. B., \& Kloep, M. (2002). Lifespan development: Resources, challenges and risks. London, England: Thomson Learning. Retrieved from https://books.google.co.nz/

Hochschild, A. R. (1983). The managed heart: Commercialization of human feeling. Berkeley, CA: University of California Press.

Huyton, J. (2009). Significant personal disclosure: Exploring the support and development needs of HE tutors engaged in the emotion work associated with supporting students. Journal of Learning Development in Higher Education (1), 1-18. 
Kelloway, E. K. (2017). Toward evidence-based practice in organizational wellbeing. In C. L. Cooper \& M. P. Leiter (Eds.), The Routledge companion to wellbeing at work (pp. 7082). New York, NY: Routledge.

Kloep, M., Hendry, L., \& Saunders, D. (2009). A new perspective on human development. In Conference of the International Journal of Arts and Sciences (Vol. 1, No. 6, pp. 332-343). Retrieved from https://pdfs.semanticscholar.org/9f30/4cc2ec19dfa4f88c29e6e8c9843511673010.pdf

Layous, K. (2018). Malleability and intentional activities. In E. Diener, S. Oishi, \& L. Tay (Eds.), Handbook of well-being. Salt Lake City, UT: DEF Publishers. doi: nobascholar.com

Leiter, M. P., \& Cooper, C. L. (2017). The state of the art of workplace wellbeing. In C. L. Cooper \& M. P. Leiter (Eds.), The Routledge companion to wellbeing at work (pp. 1-10). New York, NY: Routledge.

Lent, R. W. (2004). Toward a unifying theoretical and practical perspective on well-being and psychosocial adjustment. Journal of Counseling Psychology, 51(4), 482-509. doi:10.1037/0022-0167.51.4.482

Lyubomirsky, S., \& Layous, K. (2013). How do simple positive activities increase well-being? Current Directions in Psychological Science, 22(1), 57-62. doi:10.1177/0963721412469809

Lyubomirsky, S., Sheldon, K. M., \& Schkade, D. (2005). Pursuing happiness: The architecture of sustainable change. Review of General Psychology, 9(2), 111-131. doi:10.1037/10892680.9.2.111

Malkin, C. \& Chanock, K. (2018). Academic language and learning (ALL) in Australia: An endangered or evolving species? Journal of Academic Language and Learning, 12(1), A15A32.

Manthei, R. (1997). Counselling: The skills of finding solutions to problems. Auckland, New Zealand: Addison Wesley Longman.

McCrae, R. R., \& Costa, P. T. (2003). Personality in adulthood: A five-factor theory perspective $\left(2^{\text {nd }}\right.$ ed.). New York, NY: Guilford Press.

Mitchell, C. (2008). Walking an emotional tightrope: The role of emotions in a one-to-one learning consultation. In E. Manalo, J. Bartlett-Trafford, \& S. Crozier (Eds.), Walking a tightrope: The balancing act of learning advising: Proceedings of the 2007 Annual International Conference of the Association of Tertiary Learning Advisors of Aotearoa/New Zealand (ATLAANZ) (pp. 172-184). Retrieved from http://files.eric.ed.gov/fulltext/ED518707.pdf

New Zealand Union of Students' Associations. (2018). Kei te pai? Report on student mental health in Aotearoa. Retrieved from https://gallery.mailchimp.com/b109fde7924adea2d9afaa28d/files/ad0db517-d37f-4075-99846236b5838f0d/Kei_Te_Pai_Mental_Health_Report_Main_.pdf

Ng, T. W. H., \& Sorensen, K. L. (2009). Dispositional affectivity and work-related outcomes: A meta-analysis. Journal of Applied Social Psychology, 39(6), 1255-1287. doi:10.1111/j.15591816.2009.00481.x 
O'Toole, V., Ogier-Price, A., \& Hucks, A. (2010). Investigation into the role of emotions in tertiary teaching: A pilot study into the emotions experienced by tertiary educators in their teaching contexts: Final report. Retrieved from

https://akoaotearoa.ac.nz/download/ng/file/group-7/investigation-into-the-role-of-emotionsin-tertiary-teaching.pdf

Ryan, R. M., \& Deci, E. L. (2001). On happiness and human potentials: A review of research on hedonic and eudaimonic well-being. Annual Review of Psychology, 52(1), 141-166. doi:10.1146/annurev.psych.52.1.141

Ryff, C. D. (1989). Happiness is everything, or is it? Explorations on the meaning of psychological well-being. Journal of Personality and Social Psychology, 57(6), 1069-1081. doi:10.1037/0022-3514.57.6.1069

Schaufeli, W. B., \& Bakker, A. B. (2004). Job demands, job resources, and their relationship with burnout and engagement: A multi-sample study. Journal of Organizational Behavior, 25, 293-315.

Scheier, M. F., \& Carver, C. S. (1985). Optimism, coping, and health: Assessment and implications of generalized outcome expectancies. Health Psychology, 4(3), 219-247.

Seligman, M. E. P. (2011). Flourish: A visionary new understanding of happiness and well-being. New York, NY: Free Press.

Siegrist, J. (1996). Adverse health effects of high-effort/low-reward conditions. Journal of Occupational Health Psychology, 1(1), 27.

Sin, N. L., \& Lyubomirsky, S. (2009). Enhancing well-being and alleviating depressive symptoms with positive psychology interventions: A practice-friendly meta-analysis. Journal of Clinical Psychology: In Session, 65(5), 467-487. doi:10.1002/jclp.20593

Skinner, E. A., Edge, K., Altman, J., \& Sherwood, H. (2003). Searching for the structure of coping: A review and critique of category systems for classifying ways of coping. Psychological Bulletin, 129(2), 216-269. doi:10.1037/0033-2909.129.2.21

Sotardi, V.A. \& Friesen, M.D. (2017). Student reflections on learning and studying during the transition to university: Implications for tertiary learning advisors. ATLAANZ Journal, 2(1), $19-35$.

Staw, B. M., \& Cohen-Charash, Y. (2005). The dispositional approach to job satisfaction: more than a mirage, but not yet an oasis. Journal of Organizational Behavior, 26(1), 59-78. doi:10.1002/job.299

Sutton, R. E. (2004). Emotional regulation goals and strategies of teachers. Social Psychology of Education, 7, 379-398.

Thomas, R., \& Bennett, B. (2002). LAS advisers and the changing identity of the workplace: Their sources of stress and the strategies they use to cope. In Changing identities: Proceedings of the 2001 Australian Language and Academic Skills Conference. Wollongong: Learning Development, University of Wollongong. 
Warr, P. (1999). Well-being and the workplace. In D. Kahneman, E. Diener, \& N. Schwarz, (Eds.), Well-being: Foundations of hedonic psychology (pp. 392-412). New York, NY: Russell Sage Foundation.

Xanthopoulou, D., Bakker, A. B., Demerouti, E., \& Schaufeli, W. B. (2009). Reciprocal relationships between job resources, personal resources, and work engagement. Journal of Vocational Behavior, 74, 235-244. 